\title{
The ecological process of carbon cycling in terrestrial ecosystems in East Asia
}

\author{
Hiroyuki Muraoka $\cdot$ Yowhan Son $\cdot$ Jingyun Fang
}

Published online: 6 April 2010

(c) The Botanical Society of Japan and Springer 2010

Terrestrial ecosystems play crucial roles as sinks (absorbers) of atmospheric carbon dioxide $\left(\mathrm{CO}_{2}\right)$. The current state of, and future changes in, sink activity due to climate and land-use change are urgent issues to be investigated. In this context it is quite important to understand the consequences of environmental fluctuations and ecosystem processes, because these are fundamental to ecosystem functions with respect to determinants of global climate changes. There are many ways for performing the carbon cycling study of terrestrial ecosystems, including measurements of plant biomass, decomposition processes of soil organic matters and litterfall, ecophysiological processes of plants in various functional types in various vegetation types, micrometeorology and atmospheric research for interpreting vegetation functions and the ecosystem-scale $\mathrm{CO}_{2}$ budget, remote sensing of ecosystem structure, and simulation modeling. These intensive researches from plot to regional scales have contributed to our present knowledge of the global carbon budget and its effects on the interactions between the biosphere and climate system. However, we still come up against many

H. Muraoka ( $\square)$

Institute for Basin Ecosystem Studies, Gifu University,

1-1 Yanagido, Gifu 501-1193, Japan

e-mail: muraoka@green.gifu-u.ac.jp

Y. Son

Division of Environmental Science and Ecological Engineering,

Korea University, Anam-dong, Sungbuk-ku,

Seoul 136-701, Korea

J. Fang

Department of Ecology, College of Urban and Environmental

Sciences, Peking University, Beijing 100871, China uncertainties in the study of ecosystem function and in its temporal and spatial dynamics as well. Taking into account these uncertainties and issues, and in order to estimate the carbon budget in an ecosystem, ecosystem ecology (including micrometeorological aspects) is intended to measure those processes involved in a system and their fluctuations. The study also aims to find the ecosystems' response to or constraint by the climate and in the vegetation structure itself. Understanding of these factors promises better prediction of climate-change effects on ecosystems as well as the feedback effects of ecosystems on climate.

This special issue features the current understanding of the ecological and micrometeorological processes involved in forest, grassland, and agricultural ecosystems. The A3 Foresight Program "Quantifying and predicting terrestrial carbon sinks in East Asia: toward a network of climate change research," supported jointly by the Japan Society for the Promotion of Science (JSPS), the National Research Foundation of Korea (NRF), and the National Natural Science Foundation of China (NSFC), aims to build a collaborative research and education platform focusing on the ecological processes in carbon cycling in terrestrial ecosystems in East Asia. This project has two major research objectives: (1) to measure and evaluate the distribution of ecosystems and the temporal dynamics of internal carbon cycling in individual systems, and (2) to reveal the temporal and spatial dynamics of biotic and abiotic factors controlling carbon cycling in individual ecosystems. These two aspects are tightly linked, because the mechanisms of ecosystem stability or succession are highly dependent on the interaction of structure, function, and matter cycling in the system.

We express our appreciation to the editorial team of the Journal of Plant Research for giving us the opportunity to 
organize this special issue. We sincerely hope our papers will lead to bridging ecosystem ecology and plant science.

Project leaders of the A3 Foresight Program

Hiroyuki Muraoka (Gifu University, Japan)

Yowhan Son (Korea University, Korea)

Jingyun Fang (Peking University, China)

\section{Editors of the special issue}

Hiroyuki Muraoka (Gifu University, Japan)

Jin-Sheng He (Peking University, China) 